\title{
Características das funções orais de indivíduos com má oclusão Classe III e diferentes tipos faciais
}

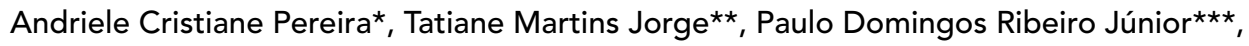

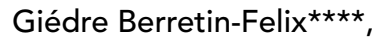

\begin{abstract}
Resumo
Objetivos: o presente estudo pretendeu investigar se o padrão facial acarreta implicações no desempenho das funções de respiração, mastigação, deglutição e fala em indivíduos com má oclusão Classe III esquelética. Metodologia: analisaram-se os dados da avaliação de 40 pacientes adultos com má oclusão Classe III, sendo 25 dolicocefálicos e 15 mesocefálicos. Consideraram-se aspectos relacionados à postura habitual de lábios e língua, modo respiratório, tipo mastigatório, padrão de deglutição e fala. Resultados: a postura alterada de lábios foi significantemente maior para o grupo de dolicocefálicos, enquanto a postura de língua no assoalho da boca foi predominante em ambos os grupos. Quanto ao modo respiratório, verificou-se que o modo oral ou oro-nasal foi significantemente maior para o grupo de dolicocefálicos. A mastigação mostrou-se alterada para ambos os grupos, enquanto a interposição de língua durante a deglutição predominou no grupo mesocefálico e a anteriorização de língua na fala no dolicocefálico. A interposição de língua na deglutição mostrou-se relacionada com a ocorrência de anteriorização de língua na fala. Conclusões: o tipo dolicocefálico contribui para a ocorrência de alterações na postura habitual dos lábios, bem como nas funções de respiração e fala, enquanto o tipo mesocefálico influenciou as características de deglutição de indivíduos com má oclusão Classe III esquelética.
\end{abstract}

Palavras-chave: Má oclusão de Angle Classe III. Respiração. Mastigação. Deglutição. Fala.

\section{INTRODUÇÃO}

O sistema estomatognático identifica um conjunto de estruturas orais que desempenham funções comuns, tendo como característica constante a participação da mandíbula. É composto pelo tecido ósseo, dentes, músculos, nervos e vasos que se relacionam de forma complexa, apesar de apresentarem biologia e estrutura funcional própria, adaptadas a funções específicas ${ }^{11}$. A relação harmoniosa entre os componentes desse sistema propicia um adequado crescimento e desenvolvimento das bases ósseas da face.

\footnotetext{
* Fonoaudióloga. Aperfeiçoamento em Fonoaudiologia e Cirurgia Ortognática pela Universidade do Sagrado Coração, Bauru/SP. Especializanda em Audiologia Clínica e Saúde do Trabalhador pelo CEFAC.

** Fonoaudióloga. Aperfeiçoamento em Fonoaudiologia e Cirurgia Ortognática pela Universidade do Sagrado Coração, Bauru/SP. Mestranda em Odontologia em Saúde Coletiva pela Faculdade de Odontologia de Bauru/ Universidade de São Paulo, Bauru/SP.

*** Professor Assistente Mestre das Disciplinas de Cirurgia e Implantodontia da graduação e pós-graduação da Faculdade de Odontologia da Universidade do Sagrado Coração, Bauru/ SP.

*** Fonoaudióloga da Clínica de Cirurgia Buco-Maxilo-Facial da Universidade do Sagrado Coração, Bauru/SP. Professora Doutora do Curso de Fonoaudiologia da Faculdade de Odontologia de Bauru/ Universidade de São Paulo, Bauru/SP.
} 
Fatores ambientais e/ou interferência em algum órgão do sistema estomatognático podem influenciar no crescimento craniofacial, alterando sua dinâmica. As desarmonias estruturais, as quais podem ser ósseas, dentárias e/ou de tecidos moles, influenciam as condições funcionais, bem como a estética facial e os aspectos psico-sociais do indivíduo ${ }^{3}$. Os indivíduos adultos portadores de desproporções maxilomandibulares possuem características miofuncionais orofaciais específicas, de acordo com o tipo de desproporção que apresentam. Estas alterações funcionais são, na verdade, adaptações, considerando-se cada padrão das bases ósseas ${ }^{4}$.

As deformidades podem ocorrer em diferentes sentidos: vertical, transversal e horizontal. No que se refere às deformidades horizontais, são comumente encontradas as alterações no desenvolvimento da mandíbula e maxila, nas quais o maior crescimento da primeira em relação à segunda resulta no prognatismo mandibular, onde há posicionamento oclusal do tipo Classe III, segundo a classificação de Angle (,20. $^{8}$.

De acordo com a literatura consultada, indivíduos com prognatismo ou Classe III de Angle apresentam as seguintes características miofuncionais: língua hipotônica e posicionada no assoalho da cavidade oral, mastigação com prevalência de movimentos mandibulares verticalizados e utilização do dorso da língua esmagando o alimento contra o palato, bem como deglutição com interposição anterior de língua ${ }^{1,18,21}$.

Outras características podem ser observadas: palato mole verticalizado, hipotonicidade do lábio inferior e hipertonia do músculo mentual, mastigação com pouca ou nenhuma ação dos músculos bucinadores, fala com inversão labial para os fonemas bilabiais e fricativos, anteriorização de língua na produção dos fonemas linguodentais, enquanto os linguoalveolares e sibilantes são produzidos com a utilização da parte média da língua ${ }^{21}$, deglutição com participação da musculatura perioral ${ }^{1,18}$ e movimentos associados de cabeça ${ }^{18}$, respiração oral habitual, disfunção temporomandibular, palato ogival, eversão do lábio inferior, lábio superior encurtado, postura habitual de lábios entreabertos, dificuldade de incisão dos alimentos com dentes anteriores ${ }^{1}$.

De acordo com as tipologias faciais, Cabrera e Enlow $^{6}$ definiram por mesofacial o tipo de face em que os vetores de crescimento e desenvolvimento facial apresentam-se em harmonia com a face, crescendo e se desenvolvendo de modo equilibrado para baixo e para frente. Já no dolicofacial existe o predomínio do crescimento vertical, ou seja, há uma predominância dos vetores de crescimento e desenvolvimento da face no sentido inferior do que no anterior. No braquifacial há um maior crescimento no sentido horizontal, onde o vetor de crescimento e desenvolvimento facial é maior no sentido anterior que no inferior, caracterizado pela musculatura forte contendo o vetor vertical.

Os indivíduos dolicofaciais ou dolicocefálicos apresentam esqueleto ósseo mais longo que as partes moles, maior tensão muscular na face, com provável mordida aberta esquelética, nariz verticalmente mais longo e com maior protusão, arco maxilar e palato duro longos, estreitos e profundos, ângulo mandibular (goníaco) aberto e base posterior do crânio mais curta. Os músculos da mastigação estão em hipofunção, o lábio superior encontra-se curto, hipotônico e funcionalmente incompetente, o inferior hipotrófico e evertido, gerando déficit de vedamento labial com significativa distância interlabial, o músculo mentual apresenta-se hipertônico na tentativa de auxiliar o vedamento labial, a língua mais anteriorizada, com dorso alto e marcas laterais. Nas funções orais de mastigação, deglutição e fala notam-se: interposição de língua e participação da musculatura perioral, articulação prejudicada quanto ao ponto articulatório dos fonemas plosivos bilabiais $(/ \mathrm{p} /$, /b/ e /m/), linguoalveolares (/t/, /d/, /1/, /n/, /s/ e $/ \mathrm{z} /$ ) e dorsais $(/ \mathrm{k} / \mathrm{e} / \mathrm{g} /$ ), sendo que os fonemas fricativos são articulados com sigmatismo (repetição viciosa do /s/ e outras sibilantes ${ }^{15}$ ). A respiração é freqüentemente oral ${ }^{9,18,21}$.

Uma vez que o fonoaudiólogo atua junto a 
pacientes com deformidades dentofaciais, nos diferentes momentos do tratamento ortodôntico-cirúrgico, é de fundamental importância que o mesmo tenha conhecimento sobre a funcionalidade do sistema estomatognático, considerando-se os diferentes padrões de bases ósseas, o que proporcionará um adequado raciocínio terapêutico para os casos.

\section{PROPOSIÇÃO}

De acordo com a literatura pesquisada, verifica-se ausência de associação entre as características morfofuncionais orofaciais segundo a classificação de Angle e o tipo facial combinados. Assim, este trabalho teve por objetivo investigar se o padrão facial acarreta implicações no desempenho das funções de respiração, mastigação, deglutição e fala em indivíduos com má oclusão Classe III de Angle.

\section{MATERIAL E MÉTODO}

Este trabalho teve início após sua aprovação pelo Comitê de Ética em Pesquisa da Universidade do Sagrado Coração (USC), sob protocolo $n^{\circ} 024 / 2004$. Vale ressaltar que todos os pacientes do Setor de Cirurgia Ortognática da referida Universidade consentiram previamente a consulta dos prontuários por meio da leitura, concordância e assinatura do Termo de Consentimento Livre e Esclarecido, o que faz parte da rotina da clínica odontológica.

A coleta de dados foi constituída da análise dos prontuários fonoaudiológicos de 40 pacientes com má oclusão Classe III esquelética, segundo classificação de Angle, sendo 26 do gênero feminino e 14 do masculino, na faixa etária de 14 a 46 anos. Dentre esses indivíduos, 15 apresentavam tipo de face mesocefálico e 25 tipo dolicocefálico. As características correspondentes à relação entre os arcos foram definidas a partir da análise das telerradiografias em norma lateral dos indivíduos. Foi considerada a presença de mordida cruzada total (MCT) e parcial (MCP), podendo ser posterior ou

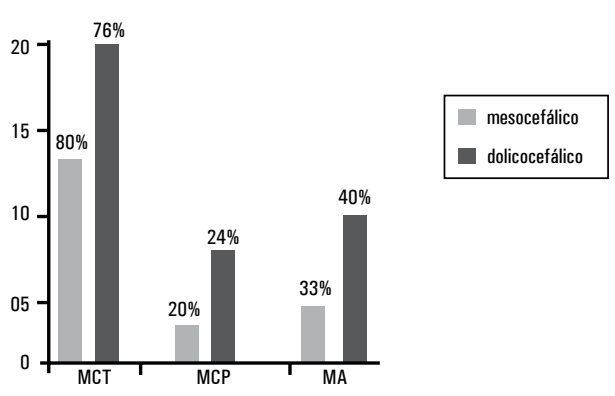

GRÁFICO 1 - Apresentação do número de indivíduos (e sua respectiva porcentagem) pertencentes a cada tipo facial, segundo a presença de mordida aberta (MA), mordida cruzada parcial (MCP) e mordida cruzada total (MCT).

anterior, bem como a presença de mordida aberta (MA) anterior ou total, as quais encontram-se apresentadas no gráfico 1 .

Durante a análise dos prontuários foram coletados os resultados da avaliação relacionados à postura habitual de língua e lábios, modo respiratório, tipo mastigatório, bem como à presença de interposição de língua e participação de músculos periorais durante a deglutição, além de desvio mandibular, anteriorização de língua e ceceio (pronúncia do /s/ e /z/ com a ponta da língua apoiando nos dentes ${ }^{16}$ ) durante a fala. Após a análise dos prontuários, os dados foram tabulados $\mathrm{e}$ encaminhados para realização do tratamento estatístico, buscando estabelecer características oromiofuncionais diferenciais entre os tipos faciais.

O tratamento estatístico para a freqüência de ocorrência foi feito utilizando-se o teste de Goodman para contrastes intra e interpopulações multinomiais. Quando o resultado estatístico mostrou significância $(\mathrm{P}<0,05)$, a conclusão foi realizada de acordo com os seguintes critérios: na comparação das ocorrências nos grupos, fixada a categoria de resposta, utilizaram-se letras minúsculas; sendo que duas proporções com uma mesma letra minúscula não diferem estatisticamente quanto às respostas dos grupos e, na comparação das categorias de respostas dentro de um dado grupo, foram utilizadas letras maiúsculas, sendo que duas proporções com uma mesma letra maiúscula não diferem estatisticamente quanto às categorias de respostas. 


\section{RESULTADOS}

Como pode ser observado na tabela 1 , a postura adequada de lábios (selados sem tensão) foi maior no grupo mesocefálico (b), ao passo que a ocorrência de postura alterada de lábios (desocluídos e selados com tensão) foi significantemente maior no grupo dolicocefálico (b). Além disso, indivíduos com Classe III mesocefálicos apresentaram predomínio de postura de lábios normal em relação ao padrão alterado (B).

Quanto à postura habitual de língua, demonstrada na tabela 2 , considerou-se adequada a postura de língua na papila palatina e como alterada a postura rebaixada ou entre dentes. Não houve diferença estatisticamente significante no que se refere à postura habitual de língua na comparação entre os grupos dolicocefálico e mesocefálico, tendo sido observado predomínio de postura alterada em relação à adequação para ambos os grupos (B).

Em relação às funções de respiração, mastigação, deglutição e fala, os resultados encontram-se descritos a seguir:

A tabela 3 mostra que indivíduos mesocefá-

Tabela 1 - Distribuição da ocorrência de respostas quanto à postura habitual dos lábios de acordo com o tipo facial.

\begin{tabular}{cccc}
\hline Tipo facial & \multicolumn{2}{c}{ Postura de lábios } & Total \\
\hline & Adequada & Alterada & \\
Mesocefálico & $13(86,67)$ b B & $2(13,33)$ a A & $15(100,00)$ \\
Dolicocefálico & $13(52,00)$ a A & $12(48,00)$ b A & $25(100,00)$ \\
\hline Teste de GOODMAN. & & &
\end{tabular}

Tabela 3 - Distribuição da ocorrência de respostas quanto ao modo respiratório de acordo com o tipo facial.

\begin{tabular}{cccc}
\hline Tipo facial & \multicolumn{2}{c}{ Modo respiratório } & Total \\
& Nasal & Alterado & \\
Mesocefálico & $13(86,67)$ b B & $2(13,33)$ a A & $15(100,00)$ \\
Dolicocefálico & $3(12,00)$ a A & $11(44,00)$ b A & $25(100,00)$ \\
\hline Teste de GOODMAN. & & &
\end{tabular}

licos apresentaram modo respiratório nasal, com diferença estatisticamente significante em relação aos dolicocefálicos (b), ao passo que o modo respiratório alterado (oral e oronasal) foi significantemente maior no grupo dolicocefálico (b). $\mathrm{O}$ grupo mesocefálico apresentou predomínio de respiração nasal em relação à oral ou oronasal (B).

Quanto ao tipo mastigatório (Tab. 4) não foram encontradas diferenças entre indivíduos mesocefálicos e dolicocefálicos, sendo que houve predomínio do tipo mastigatório alterado (unilateral ou bilateral simultâneo) em ambos os grupos (B).

A tabela 5 permite verificar que não houve diferença estatisticamente significante no que se refere à participação dos músculos periorais na comparação entre os grupos (a), ao contrário do verificado para a interposição de língua durante a deglutição, cuja ocorrência foi significantemente maior para o grupo mesocefálico (b). No entanto, observou-se predomínio de ambos os aspectos estudados para os diferentes grupos, comparativamente à ausência de participação da musculatura perioral e ausência de interposição de língua (B).

Com relação à fala, pode-se observar na tabela 6

Tabela 2 - Distribuição da ocorrência de respostas quanto à postura habitual da língua segundo o tipo facial.

\begin{tabular}{cccc}
\hline Tipo facial & \multicolumn{2}{c}{ Postura de língua } & Total \\
\hline & Adequada & Alterada & \\
Mesocefálico & $1(6,67)$ a A & $14(93,33)$ a B & $15(100,00)$ \\
Dolicocefálico & $3(12,00)$ a A & $22(88,00)$ a B & $25(100,00)$ \\
\hline Teste de GOODMAN. & & &
\end{tabular}

Tabela 4 - Distribuição da ocorrência de respostas quanto ao tipo de mastigação de acordo com o tipo facial.

\begin{tabular}{cccc}
\hline Tipo facial & \multicolumn{2}{c}{ Tipo de mastigação } & Total \\
\hline & $\begin{array}{c}\text { Bilateral } \\
\text { Alternada }\end{array}$ & Alterada \\
Mesocefálico & $3(20,00)$ a A & $12(80,00)$ a B & $15(100,00)$ \\
Dolicocefálico & $8(32,00)$ a A & $17(68,00)$ a B & $25(100,00)$ \\
\hline Teste de GOODMAN & & &
\end{tabular}


que não houve diferença estatisticamente significante entre os grupos, no que diz respeito à presença de desvio mandibular (a). Porém, ao analisar a ocorrência deste aspecto, para cada grupo estudado, foi possível verificar que a ausência de desvio mandibular foi predominante em relação à presença (B).

Os resultados da tabela 7 demonstram que o grupo dolicocefálico apresentou maior ocorrência de anteriorização de língua durante a fala em relação ao mesocefálico (b), enquanto que para o ceceio não houve diferença entre os grupos. Quando comparados os grupos entre si constatou-se ocor- rência significantemente maior de anteriorização de língua e ceceio durante a fala no grupo dolicocefálico em relação à ausência dessas alterações (B).

$\mathrm{Na}$ tabela 8 pode-se observar que, tanto para a anteriorização de língua como para a presença de ceceio durante a fala em relação à presença de interposição de língua durante a deglutição, não foram encontradas diferenças estatisticamente significantes entre os grupos (a). Por outro lado, na presença de interposição de língua durante a deglutição houve predomínio de ocorrência de anteriorização de língua na fala e ceceio em relação à ausência dessas alterações (B).

Tabela 5 - Distribuição da ocorrência de respostas quanto à participação dos músculos $(\mathrm{mm})$ periorais e interposição de língua durante a deglutição de acordo com o tipo facial.

\begin{tabular}{|c|c|c|c|c|}
\hline \multirow[t]{2}{*}{ Tipo facial } & \multicolumn{2}{|c|}{ Interposição de língua } & \multicolumn{2}{|c|}{ Participação dos mm. periorais } \\
\hline & Ausente & Presente & Ausente & Presente \\
\hline Mesocefálico & $1(6,67)$ a $A$ & $14(93,33)$ a B & $3(20,00)$ a $A$ & $12(80,00)$ a $B$ \\
\hline Dolicocefálico & $8(32,00)$ b A & $17(68,00)$ a $B$ & $6(24,00)$ a $A$ & $19(76,00)$ a $B$ \\
\hline
\end{tabular}

Teste de GOODMAN.

Tabela 6 - Distribuição da ocorrência de respostas quanto ao desvio mandibular durante a fala de acordo com o tipo facial.

\begin{tabular}{cccc}
\hline Tipo facial & \multicolumn{2}{c}{ Desvio mandibular fala } & Total \\
\hline & Ausente & Presente & Ausente \\
Mesocefálico & $14(93,33)$ a B & $1(6,67)$ a A & $15(100,00)$ \\
Dolicocefálico & $21(84,00)$ a B & $4(16,00)$ a A & $25(100,00)$ \\
\hline
\end{tabular}

Teste de GOODMAN.

Tabela 7 - Distribuição da ocorrência de respostas quanto à anteriorização de língua e ceceio durante a fala de acordo com 0 tipo facial.

\begin{tabular}{|c|c|c|c|c|}
\hline \multirow[t]{2}{*}{ Tipo facial } & \multicolumn{2}{|c|}{ Anteriorização de língua } & \multicolumn{2}{|c|}{ Ceceio } \\
\hline & Ausente & Presente & Ausente & Presente \\
\hline Mesocefálico & $7(46,67)$ b A & $8(53,33)$ a $A$ & $6(40,00)$ a $A$ & $9(60,00)$ a $A$ \\
\hline Dolicocefálico & $4(16,00)$ a $A$ & $21(84,00)$ b B & $8(32,00)$ a $A$ & $17(68,00)$ a $B$ \\
\hline
\end{tabular}

Teste de GOODMAN. 
Características das funções orais de indivíduos com má oclusão Classe III e diferentes tipos faciais

Tabela 8 - Distribuição da ocorrência de respostas quanto à anteriorização de língua e ceceio durante a fala de acordo com a interposição de língua durante a deglutição.

\begin{tabular}{|c|c|c|c|c|}
\hline \multirow[t]{2}{*}{$\begin{array}{l}\text { Interposição de língua na } \\
\text { deglutição }\end{array}$} & \multicolumn{2}{|c|}{ Anteriorização de língua na fala } & \multicolumn{2}{|c|}{ Ceceio } \\
\hline & Ausente & Presente & Ausente & Presente \\
\hline Mesocefálico & $3(33,33)$ a $A$ & $6(66,67)$ a $A$ & $3(33,33)$ a $A$ & $6(66,67)$ a $A$ \\
\hline Dolicocefálico & $8(25,81)$ a $A$ & $23(74,19)$ a $B$ & $11(35,48)$ a $A$ & $20(64,52)$ a $B$ \\
\hline
\end{tabular}

Teste de GOODMAN.

\section{DISCUSSÃO}

Os padrões miofuncionais orofaciais de indivíduos com deformidades dentofacias vêm sendo descritos na literatura, considerando-se a relação entre os arcos dentários, porém não relacionada ao tipo facial.

Neste estudo, os indivíduos dolicocefálicos apresentaram alteração da postura habitual de lábios (lábios desocluídos e selados com tensão), ao contrário dos mesocefálicos, onde a postura de lábios apresentou-se normal (selados sem tensão), conforme demonstrado na tabela 1. Este fato pode ser justificado considerando-se que o excesso vertical ósseo, presente na face longa, dificulta e até mesmo impossibilita o vedamento labial ${ }^{4,18,21}$. Além disso, nos dolicocefálicos os músculos elevadores da mandíbula são hipotônicos, resultando em posição mandibular rebaixada, comprometendo a postura dos lábios ${ }^{1}$. Da mesma forma, os achados quanto ao modo respiratório demonstraram que indivíduos dolicocefálicos apresentam respiração oral, o que pode ter contribuído para a postura alterada de lábios.

O posicionamento habitual da língua mostrou-se alterado (rebaixado) independentemente do tipo facial, como mostra a tabela 2. De acordo com a literatura ${ }^{1,18,21}$, este achado justifica-se pela própria má oclusão Classe III, na qual o crescimento da mandíbula é maior em relação à maxila ${ }^{8,20}$, resultando em uma oclusão anteriorizada, o que favorece o posicionamento de língua alargado e rebaixado no assoalho da cavidade oral ${ }^{26}$. Portanto, a posição habitual da língua depende não apenas da dimensão vertical da cavidade oral, mas também da transversal e ântero-posterior, conforme demonstrado em exames com padronização radiográfica definida ${ }^{28}$.

A análise das funções orofaciais dos indivíduos portadores de má oclusão Classe III em relação ao tipo facial demonstrou predomínio do modo respiratório nasal nos indivíduos mesocefálicos, ao passo que nos dolicocefálicos o modo mostrou-se alterado (oral ou oronasal), como pode ser visto na tabela 3 . De acordo com a literatura ${ }^{18,21}$, a respiração é freqüentemente oral na face longa, o que pode ser explicado pelo menor espaço aéreo associado à obstrução das vias aéreas superiores ${ }^{23}$, bem como pela tendência ao crescimento vertical e diminuição do tônus muscular ${ }^{10}$. No entanto, sabese que a respiração oral acarreta alterações no crescimento craniofacial, provocando desequilíbrios morfológicos e funcionais ${ }^{29}$, bem como aumento do terço inferior da face ${ }^{13,25}$, largura craniana e facial menores, rotação do ângulo da mandíbula no sentido horário, atresia da maxila, má oclusão, entre outros ${ }^{19}$, podendo o modo respiratório ter contribuído para o tipo facial dolicocefálico em alguns indivíduos.

Com relação ao tipo de mastigação, verificouse alteração para os indivíduos com má oclusão Classe III, independentemente do tipo de face (Tab. 4). Este tipo de má oclusão resulta em movimentos verticais de mandíbula com intensa participação do dorso da língua durante o esmagamento do alimento ${ }^{7,18,21}$, pois os movimentos laterais e rotatórios da mandíbula ficam dificultados 
em decorrência da posição da maxila em relação à mandíbula ${ }^{18}$. Uma das conseqüências dessa característica observada é a dificuldade na trituração de alimentos, que acarreta um déficit na eficiência mastigatória ${ }^{12}$. Além disso, a alteração do modo respiratório nos dolicocefálicos pode ter contribuído para o comprometimento do padrão mastigatório $^{29}$, assim como a presença de mordida cruzada verificada em ambos os grupos estudados, pode ter resultado em mastigação do lado cruzado ${ }^{18}$.

No que se refere à função de deglutição, verificou-se ocorrência de interposição de língua significantemente maior para o grupo de indivíduos mesocefálicos (Tab. 5) em relação aos dolicocefálicos. Porém, ambos os grupos apresentaram predomínio desta característica durante a deglutição. A interposição de língua durante a deglutição pode ser resultante da má oclusão Classe III ${ }^{4,16}$, da presença de mordida aberta ${ }^{16}$, do tamanho desproporcional da língua em relação à cavidade oral, da hipotensão da língua, da ocorrência de hábitos orais deletérios, bem como da respiração oral ou oronasal ${ }^{7}$. De acordo com os achados deste estudo, era esperado encontrar predomínio de interposição de língua durante a deglutição nos dolicocefálicos, uma vez que os mesmos apresentaram maior ocorrência de respiração oral, assim como maior porcentagem de mordida aberta. Assim, parece que, para os indivíduos da amostra, a presença de hábitos e as condições da musculatura da língua (não consideradas nesse estudo), bem como seu posicionamento habitual, semelhante em ambos os grupos, foram determinantes nesse aspecto.

Outra característica observada quanto à deglutição diz respeito à participação exagerada da musculatura perioral para os indivíduos com má oclusão Classe III, que ocorreu de modo predominante, independentemente do tipo de face (Tab. 5). Esta característica de deglutição tem como causa mais provável a hipotonicidade dos órgãos fonoarticulatórios e projeção de língua ${ }^{7}$, tendo sido observada maior ocorrência desta última no grupo mesocefálico. Além disso, o padrão facial, especialmente a face longa, pode ter contribuído para tal ocorrência ${ }^{5}$. Assim, pode-se considerar que ambos os tipos de face estudados contribuem para a ocorrência da participação da musculatura perioral.

A fala foi analisada quanto aos seguintes aspectos: desvio mandibular, anteriorização de língua e ceceio, relacionados ao tipo facial. Além disso, analisou-se a relação entre interposição de língua durante a deglutição e anteriorização de língua e ceceio durante a fala.

No que diz respeito ao desvio mandibular (Tab. 6) não houve diferença estatisticamente significante entre os grupos considerados. Verificouse, ainda, predomínio de ausência deste aspecto, para cada grupo estudado. De acordo com a literatura, o desvio mandibular durante a fala pode ser devido à presença de contatos prematuros, interferências oclusais ${ }^{2}$, respiração oral, mordida cruzada lateral ou aberta anterior e a quadros de disfunção craniomandibular (DCM) ${ }^{22}$. Vale considerar que todos esses aspectos poderiam estar presentes, de modo isolado ou combinado, nos indivíduos desse estudo, não sendo possivel, nesta pesquisa, demonstrar tal relação.

Outro aspecto investigado diz respeito à anteriorização de língua durante a fala, com relação aos tipos faciais (Tab. 7). Verificou-se predomínio desta alteração para o grupo de indivíduos dolicocefálicos, conforme achados anteriores ${ }^{24}$. É importante ressaltar que, neste estudo, os dolicocefálicos apresentaram maior porcentagem de mordida aberta, o que está relacionado às causas prováveis de anteriorização de língua durante a fala ${ }^{16,22}$, além da relação molar Classe III $^{3}$.

A investigação quanto à presença de ceceio durante a fala não indicou diferença significante entre os grupos estudados (Tab. 7). Os indivíduos com má oclusão Classe III, para compensar as alterações estruturais e funcionais que apresentam, podem manifestar alterações na articulação dos sons da fala ${ }^{26}$. No entanto, é importante considerar que, no grupo de indivíduos dolicocefálicos, houve 
maior ocorrência de anteriorização de língua e de ceceio na fala em relação à ausência dessas alterações. Nesse sentido, o ceceio pode estar relacionado à presença de mordida aberta, comumente presente em dolicocefálicos ${ }^{22}$, bem como à respiração oral ${ }^{29}$.

Com relação à anteriorização de língua e ceceio durante a fala em comparação à presença de interposição de língua na deglutição (Tab. 8), verificou-se relação significante entre ambos os aspectos. Esses achados concordam com os de outros autores ${ }^{14,27}$, os quais relatam que a anteriorização da língua durante a produção dos fonemas linguodentais está freqüentemente associada a um padrão de deglutição semelhante, ou seja, com interposição de língua. Da mesma forma, a deglutição com interposição de língua decorrente da mordida aberta anterior está relacionada à presença de transtornos na fala ${ }^{17}$.

\section{CONCLUSÃO}

De acordo com os dados obtidos podemos concluir que o padrão facial influenciou os achados referentes às funções estomatognáticas em indivíduos com má oclusão Classe III esquelética, sendo que o tipo dolicocefálico contribui para a ocorrência de alterações na postura habitual dos lábios, bem como nas funções de respiração e fala, enquanto o tipo mesocefálico influenciou as características de deglutição de indivíduos com má oclusão Classe III esquelética.

\title{
Characteristics of the oral functions of individuals with Class III malocclusion and different facial types
}

\begin{abstract}
Aim: the present study aims at investigating whether the facial pattern may cause any alterations in the performance of breathing, mastication, deglutition and speaking functions in individuals with Class III skeletal malocclusion. Method: data from evaluation of 50 adult patients with Class III malocclusion were analyzed, of which 25 were dolicocephalic and 15 mesocephalic. Aspects related with the habitual posture of the lips and tongue, breathing manner, masticatory type, deglutition and speaking pattern were also considered. Results: the altered posture of the lips was significantly higher for the dolicocephalic group, whereas the tongue posture on the floor of the mouth was predominant in both groups considered. As for the breathing manner, it could be observed that the oral or oro-nasal manner was significantly higher for the dolicocephalic group. The presence of tongue interposition in deglutition showed itself related with the occurrence of fronting of the tongue in speech. Conclusions: the dolicocephalic type contributes towards the occurrence of alterations in the habitual lip posture as well as in the breathing and speaking functions, while the mesocephalic type has influenced the deglutition characteristics in individuals with class III skeletal malocclusion.
\end{abstract}

Key words: Malocclusion. Angle Class III. Respiration. Chewing. Deglutition. Speech.

\section{REFERÊNCIAS}

1. ALTMANN, E. B. Myofunctional therapy and orthognathic surgery. Int J Orofacial Myology, Seattle, v. 13, no. 3, p. 2-12, Nov. 1987.
2. BERRETIN, G.; GENARO, K. F.; TEIXEIRA, M. L. Influência da desordem craniomandibular sobre a fala. Revista CEFAC, Rio de Janeiro, v. 2, n. 1, p. 23-31, 2000

3. BERRETIN-FELIX, G.; JORGE, T. M.; GENARO, K. F. Intervenção fonoaudiológica em paciente submetidos à cirurgia ortognática. In: FERREIRA, L. P.; BEFI-LOPES, D. M.; LIMONGI, S. C. O. Tratado de fonoaudiologia. São Paulo: Roca, 2004. cap. 38, p. 494-511. 
4. BIANCHINI, E. M. G. Desproporções maxilomandibulares: atuação fonoaudiológica com pacientes submetidos à cirurgia ortognática. In: MARCHESAN, I. Q. et al. (Org.). Tópicos em fonoaudiologia. São Paulo: Lovise, 1995. v. 2, cap. 8, p. 129-145.

5. BIANCHINI, E. M. G. Mastigação e ATM: avaliação e terapia. In: MARCHESAN, I. Q. Fundamentos em fonoaudiologia: aspectos clínicos da motricidade oral. Rio de Janeiro: Guanabara Koogan, 1998. cap. 5, p. 37-49.

6. CABRERA, C. A. G.; ENLOW, D.H. Crescimento e desenvolvimento craniofacial. In: CABRERA, C. A. G.; CABRERA, M. C. Ortodontia clínica. Curitiba: Produções Interativas, 1997. cap. 1, p. 1-41.

7. CATTONI, D. M. Alterações da mastigação e deglutição. In.: FERREIRA, L. P.; BEFI-LOPES, D. M.; LIMONGI, S. C. O. Tratado de fonoaudiologia, São Paulo: Roca, 2004. cap. 24, p. 277- 291.

8. COSTA, N. P. Prognatismo mandibular: avaliação cefalométrica. 1981. 81 f. Dissertação (Mestrado)-Faculdade de Odontologia, Universidade de São Paulo, São Paulo, 1981.

9. D'AGOSTINO, L. Características fonoaudiológicas nas deformidades maxilomandibulares. In: PSILLAKIS, J. M. et al. Cirurgia craniomaxilofacial: osteotomias estéticas da face. Rio de Janeiro: Medsi, 1987. cap. 32, p.317-323.

10. DI FRANCESCO, R. C. Crescimento craniofacial e distúrbios da respiração oral do ponto de vista otorrinolaringológico. In: KRAKAUER, L. H.; DI FRANCESCO, R. C.; MARCHESAN, I. Q. (Org.). Respiração oral: abordagem interdisciplinar. São José dos Campos: Pulso, 2003. cap. 3. p. 27-35.

11. DOUGLAS, C. R. Fisiologia geral do sistema estomatognático. In: Tratado de fisiologia aplicada a fonoaudiologia. São Paulo: Robe Editorial, 2002. cap. 19, p. 289-301.

12. ENGLISH, J. D.; BUSCHANG, P. H.; THROCKMORTON, G. S. Does malocclusion affect masticatory performance? Angle Orthod, Appleton, v. 72, no. 1, p. 21-27, Feb. 2002.

13. ENLOW, D. H. Crescimento facial. São Paulo: Artes Médicas, 1993.

14. FELÍCIO, C. M. Fonoaudiologia nas desordens temporomandibulares: uma ação educativa-terapêutica. São Paulo: Pancast, 1994.

15. FERREIRA, A. Novo Aurélio Século XXI: o dicionário da língua portuguesa. 3. ed. rev. e ampl. Rio de Janeiro: Nova Fronteira, 1999.

16. KASAI, R. C. B; PORTELLA, M. Q. Intervenção fonoaudiológica em pacientes submetidos ao tratamento ortodôntico cirúrgico. R Dent Press Ortodon Ortop Facial, Maringá, v. 6, n. 2, p.79-84, mar./abr. 2001
17. KHINDA, V.; GREWAL, N. Relationship of tongue-thrust swallowing and anterior open bite with articulation disorders: a clinical study. J Indian Soc Pedod Prev Dent, Chandigarh, v. 17, no. 2, p. 33-39, June 1999

18. KRAKAUER, L. H. Alteração de funções orais nos diversos tipos faciais. In: MARCHESAN, I. Q. et al. (Org.). Tópicos em fonoaudiologia. São Paulo: Lovise, 1995. v. 2, cap. 9, p. 147-154.

19. LINDER-ARONSON, S.; WOOSIDE, D. G. The growth in the sagital depth of the bony nasopharynx in relation to some other facial variables. In: McNAMARA JÚNIOR, J. A. (Ed.). Naso-respiratory function and craniofacial growth. Ann Arbor: University of Michigan, Center of human growth and development, 1979. p. 27-40. Craniofacial Growth Series, Monograph, n. 9.

20. LITTON, S. F. et al. A genetic study of class III malocclusion. Am J Orthod, St. Louis, v. 58, no. 6, p. 565-577, Dec. 1970.

21. MARCHESAN, I. Q.; BIANCHINI, E. M. G. A fonoaudiologia e a cirurgia ortognática. In: ARAÚJO, A. Cirurgia ortognática. São Paulo: Ed. Santos. 1999. cap. 26, p. 353-361.

22. MARCHESAN, I. Q. Alterações de fala de origem musculoesquelética. In.: FERREIRA, L. P.; BEFI-LOPES, D. M.; LIMONGI, S. C. O. Tratado de fonoaudiologia. São Paulo: Roca, 2004. cap. 25, p. 292-303.

23. MERIDTH, G. M. Airway and dentofacial development. Folha Med, Rio de Janeiro, v. 97, n. 1, p. 33-40, 1988.

24. PEREIRA, A. C.; JORGE, T. M.; BERRETIN-FELIX, G. Características miofuncionais orofaciais de indivíduos com má-oclusão classe III e diferentes tipos faciais. In: JORNADA FONOAUDIOLÓGICA PROFa. DR ${ }^{a}$. ALCIONE GHEDINI BRASOLOTTO, 10, 2003. Bauru. Anais... Bauru [s.n], 2003. p. 64.

25. PLANAS, P. RNO: reabilitação neuro oclusal. Rio de Janeiro: Médsi, 1998.

26. SANTOS, I. F.; PEREIRA, S. A. A prevalência de alterações de fala em indivíduos portadores de classe III. Fono atual, São Paulo, ano 4, n. 15, p. 16-21, 2001.

27. SILVERMAN, E. T. Reabilitação da fala, hábitos e terapia miofuncional nos processos restauradores. In: SEIDI, L. J. Um método dinâmico para a Odontologia restauradora. São Paulo: Panamericana, 1984. p. 650-715.

28. TESSITORE, A.; CRESPO, A. N. Análise radiográfica da posição habitual de repouso da língua. Pró-Fono Revista de Atualização Científica, Carapicuíba, v.14, n.1, p.7-16, jan./abr. 2002.

29. TESSITORE, A. Alterações oromiofuncionais em respiradores orais. In.: FERREIRA, L. P.; BEFI-LOPES, D. M.; LIMONGI, S. C. O. Tratado de fonoaudiologia, São Paulo: Roca, 2004. cap. 23, p. 261-276.
Endereço para correspondência

Andriele Cristiane Pereira

Rua Antônio dos Reis, 11-31, Jd Brasil

CEP: 17011-030 - Bauru/SP

E-mail: andriele_pereira@yahoo.com.br 